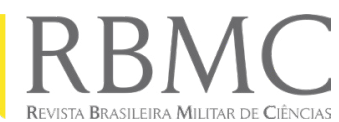

ISSN 2447-9071

doi https://doi.org/10.36414/rbmc.v7i17.90

Contato para correspondência:

Rogério José de Almeida

E-mail:

rogerio.almeida@faculdadepm.edu.br

Conflito de interesse: Não

Financiamento: Recursos próprios

Recebido: 05/04/2021

Aprovado: 19/04/2021

\section{Aspectos relacionados a empatia médica em estudantes de medicina: uma revisão integrativa}

\section{Aspects related to medical empathy in medical students: an integrative review}

Beatriz Moreira Caetano Vaz' ${ }^{1}$, Vanessa Alves Paraízo ${ }^{1}$, Rogério José de Almeida, ${ }^{1,2}$

${ }^{1}$ Pontifícia Universidade Católica de Goiás - PUC Goiás

${ }^{2}$ Faculdade da Polícia Militar - FPM

\section{Resumo}

A discussão sobre a empatia na formação médica vem se aprofundando em âmbito mundial. Escolas médicas vêm sendo investigadas e seus estudantes avaliados, principalmente por meio da Escala Jefferson de Empatia Médica versão para estudantes. Temporobjetivo analisar, pormeio de uma revisão integrativa da literatura, estudos que investigaram a empatia médica em estudantes de medicina. Trata-se de uma revisão integrativa da literatura científica com coleta dos dados nas bases PubMede Biblioteca Virtual da Saúde (BVS). Para a busca dos artigos, foram utilizados os Descritores da Saúde (DeCS):empatia, curso demedicinaeestudantes demedicina;eem inglês os Medical Subject Headings (MeSH): empathy, medicine course and medical student. Os critérios de inclusão foram: artigos que investigaram a empatia em estudantes de medicina utilizando a escala Jefferson de Empatia (versão para estudantes), publicados nos idiomas português, inglês e espanhol, durante o período de 2015 a 2020. Os critérios de exclusão foram: artigos repetidos na busca; revisão de literatura e dissertações e teses. Foram identificados um total de 17 artigos, os quais apresentaram fatores relacionados a empatia médica em estudantes de medicina, sendo: os anos de graduação, as características pessoais de cada indivíduo (gênero e idade), os fatores externos como um sistema de ensino integrado, uma matrizcurricularmais rica em conteúdo de ciências humanas e um contato prático com pacientes mais precoce, a participação em trabalhos voluntários, o contato próximo com enfermidade de amigos, as disfuncionalidades familiares, a escolha da medicina como sendo a primeira opção de carreira do estudante a a escolha de especialidades clínicas com maior contato médico-paciente. Diante das evidências encontradas é possivel entender que a empatia nos estudantes de medicina é moldada por diversos fatores durante sua formação acadêmica. Isso permite desenvolver estratégias a fim de se solucionar a tendência decrescente dos escores de empatia ao longo da graduação, por meio da introdução, principalmente, deencontro clínico mais precocee de matérias das ciências humanas nas matrizes curriculares em cursos de medicina em todo o mundo.

Palavras-Chave: Empatia; Estudantes de medicina; Relação médico-paciente;

\begin{abstract}
The discussion aboutempathy in medical education has been deepening worldwide. Medical schools around the world have been investigated and their students evaluated, mainly through the Jefferson Scale of Medical Empathy for students. This study aims to analyze, through an integrative review of the scientific literature worldwide, results of studies that investigated empathy in medical students. It is an integrative review of the scientific literature with data collection in the PubMed and Virtual Health Library (VHL) databases. To search for the articles, the Health Descriptors (DeCS) were used: empathy, medical school, and medical students; and in English the Medical Subject Headings (MeSH):empathy, medicine course and medical student. The inclusion criteria were: articles that investigated empathy in medical students using the Jefferson Empathy Scale (version for students), published in Portuguese, English, and Spanish, during the period from 2015 to 2020. The exclusion criteria were: repeated articles
\end{abstract}


in the search; literature review and dissertations and theses. A total of 17 articles were identified, which presented factors related to medical empathy in medical students, being: the influence of undergraduate years, the personal characteristics of each individual (gender and age), external factors such as an integrated education system, a curriculum class richer in humanities content and earlier practical contact with patients, participation in volunteer work, close contact with the illness of friends, family dysfunctionalities, choosing medicine as the student's first career option and choosing clinical specialties with greater doctor-patient contact. Given the evidence found, it is possible to understand that empathy in medical students is shaped by several factors during their academic training. This allows the strategy to be developed in order to resolve the decreasing trend in empathy scores throughout undergraduate courses, through the introduction, mainly, of an earlierclinical encounterand of human sciences in curricular classes in medical courses worldwide.

Keywords: Empathy; Medical students; Doctor-patient relationship.

\section{Introdução}

A criação do Relatório Flexner em 1910, que propunha a fragmentação das disciplinas e a divisão do ensino em ciclos básico e profissionalizante, representou um grande avanço e, ao mesmo tempo, a criação de novos problemas para a formação médica em todo o mundo. Sua proposta era de um ensino hospitalocêntrico, biologicista, com ênfase em especializações e disciplinas fracionadas, deixando a metodologia de ensino pouco flexível, centrada no professor e aquém das necessidades sociais que logo transformariam o modelo de assistência em saúde. A formação médica era de um profissional individualista, especialista, com uma visão segmentada do paciente e que se fundamentava na patogenia e na terapêutica, esquecendo-se de outros aspectos que englobam o processo saúde-doença'?

O modelo biomédico proposto tornou-se incompatível com a realidade que assumiu a saúde brasileira. Desse modo, em 2001 foram elaboradas as primeiras Diretrizes Curriculares Nacionais (DCN) para o curso de medicina, que visavam um ensino interdisciplinar, centrado no aluno e que buscasse a formação integral do estudante, sempre articulando ensino, pesquisa e extensão². Em 2014, as novas DCN reiteraram a participação ativa do acadêmico e trouxeram a importância dos aspectos humanísticos e socioculturais na prática clínica ${ }^{3}$. Assim, surgiu um novo padrão de"médico ideal": generalista, crítico, reflexivo, ético, empático, capaz de realizar ações de prevenção, promoção e proteção à saúde, sempre respeitando a dignidade humana4 .

A relação médico/estudante-paciente vai muito além do encontro situacional, da anamnese, do exame físico e das condutas médicas tomadas. Trata-se de uma interação que envolve confiança e responsabilidade, caracterizada por compromissos e deveres de ambos os atores. Nesse sentido, a empatia se torna uma ferramenta muito importante na prática clínica, uma vez que possui papel significativo para o desenvolvimento de habilidades cognitivas, afetivas e sociais ${ }^{5}$. Trata-se de uma consciência do sujeito na imaginação sobre a emoção das outras pessoas ${ }^{5}$.

A empatia é um conjunto de atitudes que um indivíduo emite diante uma interação social, cuja principal função é a manutenção de uma relação saudável com base na compreensão e na expressão dos sentimentos envolvidos. Para o ser humano se desenvolver de forma harmônica é necessário o desempenho de habilidades sociais empáticas. Esse desempenho pode ser adquirido a partir de treinamentos durante a formação social e acadêmica dos indivíduos ${ }^{6}$.

A empatia na relação médico-paciente destaca-se como um elemento central nesse contexto, trazendo benefícios para ambos. O cuidado empático envolve o enaltecimento da equidade em saúde, em que há uma valorização recíproca entre os envolvidos. Isso possibilita uma maior satisfação dos pacientes quanto ao atendimento, e amplia a habilidade diagnóstica por parte dos médicos, contribuindo também para a implementação do melhor tratamento e para a diminuição do risco de erros desse profissional?.

Apesar de a empatia ser uma característica central nessa relação, seus baixos níveis nos profissionais médicos são documentados e discutidos na literatura científica. Esse fato pode ter relação direta com a formação acadêmica em medicina, período decisivo para o desenvolvimento da empatia médica ${ }^{8}$. Dentre os fatores associados a baixa empatia nos cursos de medicina estão: alta carga horária do curso, estresse cotidiano, pouca interação familiar, menos tempo dedicado a atividades culturais e de lazer e atitudes negativas de professores e preceptores ${ }^{9}$. Já fatores positivos que impactam na empatia são: ser de sexo feminino, ausência de doença pessoal, morar em residência própria e menor idade ${ }^{8}$.

Assim, o presente estudo teve por objetivo identificar, por meio de uma revisão integrativa da literatura científica, estudos que investigaram a empatia em estudantes de medicina. 


\section{Métodos}

Trata-se de uma revisão integrativa da literatura científica, que se configura como a busca por unir o conhecimento atual sobre uma temática específica, de forma a incluir uma variedade mais ampla de estudos, que outras revisões não permitem, abrangendo estudos experimentais e não-experimentais no levantamento de dados. Visa auxiliar na visão crítica do que está sendo publicado cientificamente, apresentando assim, uma visão mais ampla dos dados científicos publicados ${ }^{10}$.

O problema de pesquisa utilizado para a busca na literatura foi: Como se configura a empatia em estudantes de medicina nas diversas escolas médicas do mundo? Assim, para a coleta dos dados foram utilizadas as bases de dados PubMed e Biblioteca Virtual da Saúde (BVS). Para a busca dos artigos, foram utilizados em português os Descritores da Saúde (DeCS): empatia, curso de medicina e estudantes de medicina; e em inglês os Medical Subject Headings (MeSH): empathy, medicine course and medical student. Durante a busca, empregaram-se os operadores booleanos "AND" e "OR" entre os termos utilizados visando alcançar produção bibliográfica específica.

Os critérios de inclusão foram: artigos que investigaram a empatia em estudantes de medicina utilizando a escala Jefferson de Empatia (versão para estudantes); publicados nos idiomas português, inglês e espanhol, durante o período de 2015 a 2020. Os critérios de exclusão foram: artigos repetidos na busca; de revisão de literatura; dissertações e teses.

A busca nas bases de dados ocorreu em junho de 2020. Os artigos foram selecionados segundo os critérios do Preferred Reporting Items for Systematic Reviews and Meta-Analyses $(P R I S M A)^{11}$. A partir da pergunta norteadora seguiu-se uma seleção sistematizada e quatro etapas, sendo elas: Identificação, Seleção, Elegibilidade E Inclusão. A busca foi realizada de forma independente por duas pesquisadoras e foram confrontados os resultados para se chegar a amostra final.

Foi realizada a análise crítica dos estudos incluídos. Nesta fase foram organizadas as características dos estudos, com avaliação dos métodos e resultados. Em seguida, realizou-se a interpretação dos estudos e uma síntese dos resultados, com a comparação dos dados selecionados. O procedimento metodológico desse estudo é descrito no fluxograma que segue (Figura 1):
Figura 1. Fluxograma baseado no modelo PRISMA com os resultados da seleção de artigos que investigaram a empatia em estudantes de medicina utilizando a escala Jefferson de Empatia (versão para estudantes).

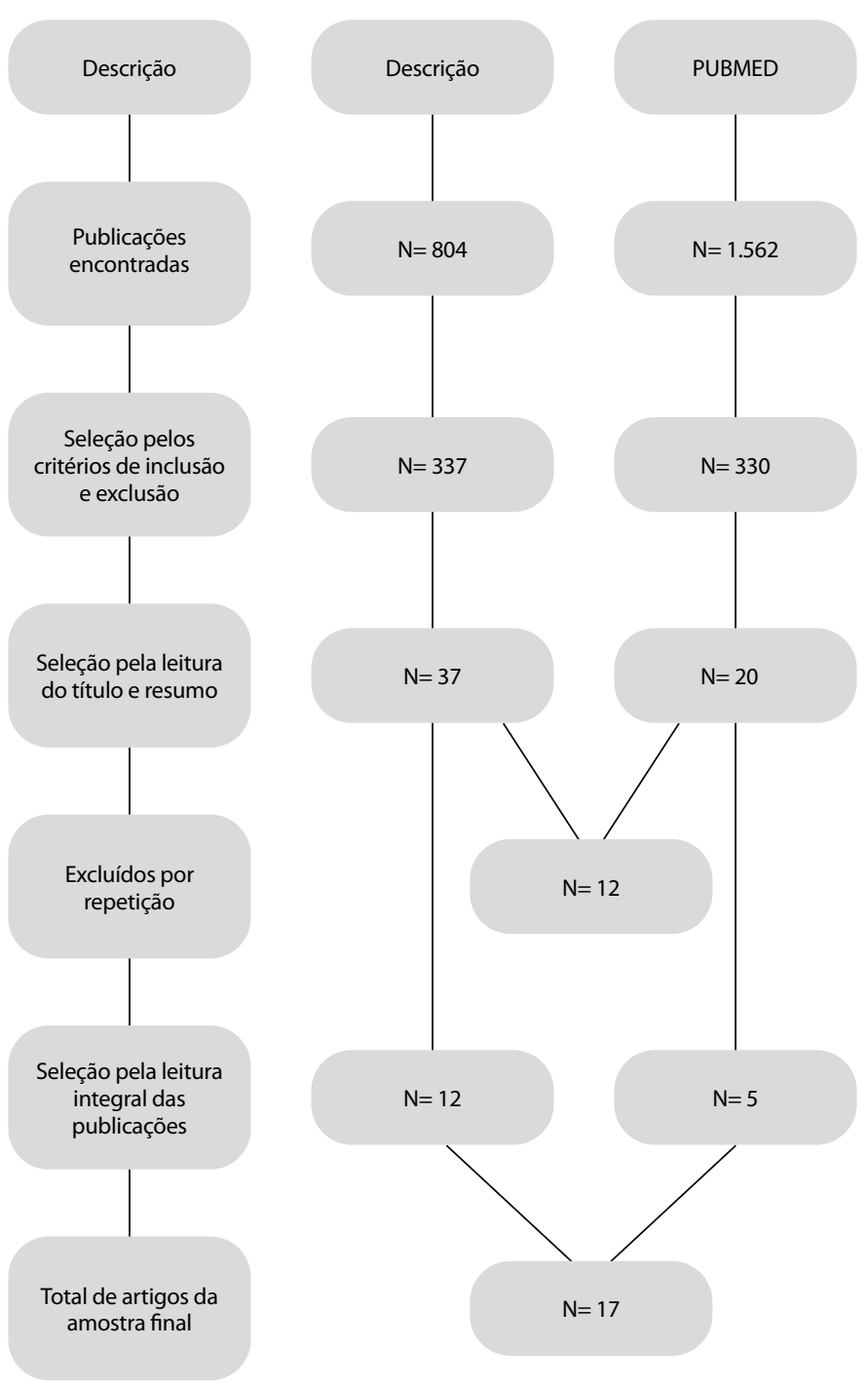

Fonte: Elaborada pelos autores.

Foram encontrados 17 artigos, publicados entre 2015 e 2020, todos em periódicos de medicina, conforme detalhado no Quadro 1. Dentre eles, incluem-se dois estudos multicêntricos que comparam estudantes de nacionalidades diferentes, sendo um realizado em universidades do Reino Unido, Nova Zelândia e Irlanda, e o outro em universidades do Equador e Colômbia. As demais pesquisas foram conduzidas nos respectivos países: Brasil (2), África do Sul (1), Argentina (1), China (2), Colômbia (1), Estados Unidos (1), Índia (1), Irã (1), Iraque (1) Irlanda (1), 
Paquistão (2), Portugal (1). Dessa forma, a maioria dos estudos foram realizados com estudantes de medicina dos continentes asiático e americano.

Quadro 1. Características dos artigos que investigaram a empatia em estudantes de medicina utilizando a escala Jefferson de Empatia (versão para estudantes), em ordem crescente do ano de publicação.

\begin{tabular}{|c|c|c|c|}
\hline $\begin{array}{l}\text { Autores e ano de } \\
\text { publicação }\end{array}$ & Título & Periódico & Tipo de estudo \\
\hline $\begin{array}{l}\text { Anaya MVM, Ama- } \\
\text { dor LRT, Martínez } \\
\text { FG. (2015)12 }\end{array}$ & $\begin{array}{l}\text { Factores relaciona- } \\
\text { dos con la empatía } \\
\text { en estudiantes } \\
\text { de medicina de } \\
\text { la Universidad de } \\
\text { Cartagena }\end{array}$ & $\begin{array}{l}\text { Revista Clínica } \\
\text { de Medicina de } \\
\text { Família }\end{array}$ & $\begin{array}{l}\text { Estudo transver- } \\
\text { sal com estudan- } \\
\text { tes de Medicina } \\
\text { da Universidade } \\
\text { de Cartagena. }\end{array}$ \\
\hline $\begin{array}{l}\text { Benabbas R. } \\
(2016) 13\end{array}$ & $\begin{array}{l}\text { Empathy in Iranian } \\
\text { medical students: } \\
\text { A comparison by } \\
\text { age, gender, aca- } \\
\text { demic performan- } \\
\text { ce and specialty } \\
\text { preferences }\end{array}$ & $\begin{array}{l}\text { Medical Journal } \\
\text { of the Islamic } \\
\text { Republic of Iran } \\
\text { (MJIRI) }\end{array}$ & $\begin{array}{l}\text { Estudo transver- } \\
\text { sal com alunos } \\
\text { da escola de } \\
\text { medicina da } \\
\text { Universidade de } \\
\text { Ciências Médicas } \\
\text { do Irã. }\end{array}$ \\
\hline $\begin{array}{l}\text { Quince TA, Kin- } \\
\text { nersley P, Hales J, } \\
\text { Silva A, Moriarty H, } \\
\text { Thiemann P, et al. } \\
\text { (2016)14 }\end{array}$ & $\begin{array}{l}\text { Empathy among } \\
\text { undergraduate } \\
\text { medical students: } \\
\text { A multi-centre } \\
\text { cross-sectional } \\
\text { comparison of stu- } \\
\text { dents beginning } \\
\text { and approaching } \\
\text { the end of their } \\
\text { course }\end{array}$ & $\begin{array}{l}\text { BMC Medical } \\
\text { Education }\end{array}$ & $\begin{array}{l}\text { Estudo transver- } \\
\text { sal multicêntrico. }\end{array}$ \\
\hline $\begin{array}{l}\text { Raof AM, Yassin BA. } \\
(2016) 15\end{array}$ & $\begin{array}{l}\text { Measuring Empa- } \\
\text { thy Levels among } \\
\text { Kurdish Medical } \\
\text { Students in Erbil } \\
\text { City, Iraq }\end{array}$ & $\begin{array}{l}\text { Sultan Qaboos } \\
\text { University Medi- } \\
\text { cal Journal }\end{array}$ & $\begin{array}{l}\text { Estudo transver- } \\
\text { sal com estudan- } \\
\text { tes de medicina } \\
\text { do Iraque. }\end{array}$ \\
\hline $\begin{array}{l}\text { Calzadilla-Núñez } \\
\text { A, Díaz-Narváez } \\
\text { VP, Dávila-Pontón } \\
\text { Y, Aguilera-Muños } \\
\text { J, Fortich-Mesa N, } \\
\text { Aparicio-Marenco D, } \\
\text { et al. (2017)16 }\end{array}$ & $\begin{array}{l}\text { Erosión empática } \\
\text { durante la forma- } \\
\text { ción médica según } \\
\text { el género. Estudio } \\
\text { transversal }\end{array}$ & $\begin{array}{l}\text { Archivos Argenti- } \\
\text { nos de Pediatria }\end{array}$ & $\begin{array}{l}\text { Estudo transver- } \\
\text { sal com alunos } \\
\text { de Medicina } \\
\text { do primeiro ao } \\
\text { sexto ano da } \\
\text { Universidad del } \\
\text { Azuay (Cuenca, } \\
\text { Ecuador) e da } \\
\text { Corporación Uni- } \\
\text { versitaria Rafael } \\
\text { Núnez (Cartage- } \\
\text { na, Colombia). }\end{array}$ \\
\hline
\end{tabular}

\begin{tabular}{|c|c|c|c|}
\hline $\begin{array}{l}\text { Chatterjee A, Ravi- } \\
\text { kumar R, Singh S, } \\
\text { Chauhan OS, Goel } \\
\text { M. (2017)17 }\end{array}$ & $\begin{array}{l}\text { Clinical empathy in } \\
\text { medical students } \\
\text { in India measured } \\
\text { using the Jefferson } \\
\text { Scale of Empathy- } \\
\text { Student Version }\end{array}$ & $\begin{array}{l}\text { Journal of Educa- } \\
\text { tional Evaluation } \\
\text { for Health Profes- } \\
\text { sions }\end{array}$ & $\begin{array}{l}\text { Um estudo } \\
\text { observacional } \\
\text { transversal foi } \\
\text { conduzido entre } \\
\text { estudantes de } \\
\text { graduação em } \\
\text { medicina da Uni- } \\
\text { versity College of } \\
\text { Medical Sciences } \\
\text { e do GTB Hos- } \\
\text { pital. }\end{array}$ \\
\hline $\begin{array}{l}\text { O'Sullivan DM, } \\
\text { Moran J, Corco- } \\
\text { ran P, O'Flynn S, } \\
\text { O'Tuathaigh C, } \\
\text { O'Sullivan AM. } \\
\text { (2017)18 }\end{array}$ & $\begin{array}{l}\text { Medical school } \\
\text { selection criteria } \\
\text { as predictors of } \\
\text { medical student } \\
\text { empathy: a cross- } \\
\text {-sectional study of } \\
\text { medical students, } \\
\text { Ireland }\end{array}$ & BMJ Open & $\begin{array}{l}\text { Estudo transver- } \\
\text { sal com alunos } \\
\text { de Medicina na } \\
\text { University Colle- } \\
\text { ge Cork, Irlanda. }\end{array}$ \\
\hline $\begin{array}{l}\text { Tariq N, Rasheed T, } \\
\text { Tavakol M. (2017)19 }\end{array}$ & $\begin{array}{l}\text { A quantitative stu- } \\
\text { dy of empathy in } \\
\text { Pakistani medical } \\
\text { students: a multi- } \\
\text { centered approach }\end{array}$ & $\begin{array}{l}\text { Journal of Prima- } \\
\text { ry Care \& Com- } \\
\text { munity Health }\end{array}$ & $\begin{array}{l}\text { Estudo transver- } \\
\text { sal quantitativo } \\
\text { de } 1453 \text { alunos } \\
\text { de } 8 \text { escolas } \\
\text { médicas do } \\
\text { Paquistão, tanto } \\
\text { privadas quanto } \\
\text { estatais. }\end{array}$ \\
\hline $\begin{array}{l}\text { Li D, Xu H, Kang M, } \\
\text { Ma S. (2018)20 }\end{array}$ & $\begin{array}{l}\text { Empathy in Chi- } \\
\text { nese eight-year } \\
\text { medical program } \\
\text { students: differen- } \\
\text { ces by school year, } \\
\text { educational stage, } \\
\text { and future career } \\
\text { preference }\end{array}$ & $\begin{array}{l}\text { BMC Medical } \\
\text { Education }\end{array}$ & $\begin{array}{l}\text { Estudo transver- } \\
\text { sal com alunos } \\
\text { matriculados no } \\
\text { programa de } \\
\text { educação médica } \\
\text { de oito anos na } \\
\text { Fudan University, } \\
\text { China. }\end{array}$ \\
\hline $\begin{array}{l}\text { Moreto G, Santos IS, } \\
\text { Pessini L, Lotufo PA. } \\
\text { (2018)21 }\end{array}$ & $\begin{array}{l}\text { Assessing empathy } \\
\text { among medical } \\
\text { students: A com- } \\
\text { parative analysis } \\
\text { using two different } \\
\text { scales in a Brazilian } \\
\text { medical school }\end{array}$ & $\begin{array}{l}\text { Educación Mé- } \\
\text { dica }\end{array}$ & $\begin{array}{l}\text { Estudo trans- } \\
\text { versal. }\end{array}$ \\
\hline $\begin{array}{l}\text { Nascimento HCF, } \\
\text { Ferreira Júnior WA, } \\
\text { Silva AMTC, Car- } \\
\text { valho IGM, Bastos } \\
\text { GCFC, Almeida RJ. } \\
\text { (2018)8 }\end{array}$ & $\begin{array}{l}\text { Análise dos Níveis } \\
\text { de Empatia de } \\
\text { Estudantes de } \\
\text { Medicina }\end{array}$ & $\begin{array}{l}\text { Revista Brasileira } \\
\text { de Educação } \\
\text { Médica }\end{array}$ & $\begin{array}{l}\text { Estudo trans- } \\
\text { versal descritivo } \\
\text { com abordagem } \\
\text { quantitativa. }\end{array}$ \\
\hline $\begin{array}{l}\text { Tariq N, Tayyab A, } \\
\text { Jaffery T. (2018)22 }\end{array}$ & $\begin{array}{l}\text { Differences in } \\
\text { empathy levels of } \\
\text { medical students } \\
\text { based on gender, } \\
\text { year of medical } \\
\text { school and career } \\
\text { choice }\end{array}$ & $\begin{array}{l}\text { Journal of the } \\
\text { College of Physi- } \\
\text { cians and Surge- } \\
\text { ons Pakistan }\end{array}$ & $\begin{array}{l}\text { Estudo transver- } \\
\text { sal quantitativo } \\
\text { com os alunos } \\
\text { de Shifa College } \\
\text { of Medicine, Uni- } \\
\text { versidade Shifa } \\
\text { Tameer-e-Millat. }\end{array}$ \\
\hline
\end{tabular}




\begin{tabular}{|c|c|c|c|}
\hline $\begin{array}{l}\text { Archer E, Turner R. } \\
\text { (2019) } 23\end{array}$ & $\begin{array}{l}\text { Measuring em- } \\
\text { pathy in a group } \\
\text { of South African } \\
\text { undergraduate } \\
\text { medical students } \\
\text { using the student } \\
\text { version of the } \\
\text { Jefferson Scale of } \\
\text { Empathy }\end{array}$ & $\begin{array}{l}\text { African Journal } \\
\text { of Primary Health } \\
\text { Care \& Family } \\
\text { Medicine }\end{array}$ & $\begin{array}{l}\text { Estudo de } \\
\text { abordagem de } \\
\text { métodos mis- } \\
\text { tos, incluindo } \\
\text { qualitativos e } \\
\text { quantitativos } \\
\text { em estudantes } \\
\text { de medicina da } \\
\text { África do Sul. }\end{array}$ \\
\hline $\begin{array}{l}\text { Ulloque MJ, Villalba } \\
\text { S, Varela de Villalba } \\
\text { T, Fantini A, Quinte- } \\
\text { ros S, Díaz-Narváez } \\
\text { V. }(2019) 24\end{array}$ & $\begin{array}{l}\text { Niveles de empatía } \\
\text { en estudiantes de } \\
\text { medicina de Cór- } \\
\text { doba, Argentina }\end{array}$ & $\begin{array}{l}\text { Archivos Argenti- } \\
\text { nos de Pediatria }\end{array}$ & $\begin{array}{l}\text { Estudo transver- } \\
\text { sal com alunos } \\
\text { de medicina } \\
\text { da Universidad } \\
\text { Católica de } \\
\text { Córdoba (UCC), } \\
\text { Argentina. }\end{array}$ \\
\hline $\begin{array}{l}\text { Hojat M, Shannon } \\
\text { SC, Desantis J, Spei- } \\
\text { cher MR, Bragan } \\
\text { L, Calabrese LH. } \\
(2020) 25\end{array}$ & $\begin{array}{l}\text { Does Empathy } \\
\text { Decline in the Cli- } \\
\text { nical Phase of Me- } \\
\text { dical Education? } \\
\text { A Nationwide, } \\
\text { Multi-Institutional, } \\
\text { Cross-Sectional } \\
\text { Study of Students } \\
\text { at DO-Granting } \\
\text { Medical Schools }\end{array}$ & $\begin{array}{l}\text { Academic Me- } \\
\text { dicine }\end{array}$ & $\begin{array}{l}\text { Estudo trans- } \\
\text { versal. }\end{array}$ \\
\hline $\begin{array}{l}\text { Santiago LM, Rosen- } \\
\text { do I, Coutinho ML, } \\
\text { Maurício KS, Neto I, } \\
\text { Simões JA. (2020)26 }\end{array}$ & $\begin{array}{l}\text { Comparing em- } \\
\text { pathy in medical } \\
\text { students of two } \\
\text { Portuguese medi- } \\
\text { cine schools }\end{array}$ & $\begin{array}{l}\text { BMC Medical } \\
\text { Education }\end{array}$ & $\begin{array}{l}\text { Estudo transver- } \\
\text { sal observacional } \\
\text { com análise esta- } \\
\text { tística descritiva } \\
\text { e inferencial. }\end{array}$ \\
\hline $\begin{array}{l}\text { Ye X, Guo H, Xu Z, } \\
\text { Xiao H. (2020)27 }\end{array}$ & $\begin{array}{l}\text { Empathy variation } \\
\text { of undergraduate } \\
\text { medical students } \\
\text { after early clinical } \\
\text { contact: a cross- } \\
\text {-sectional study in } \\
\text { China }\end{array}$ & BMJ Open & $\begin{array}{l}\text { Estudo longitudi- } \\
\text { nal em estudan- } \\
\text { tes de medicina } \\
\text { da Universidade } \\
\text { de Shangai na } \\
\text { China. }\end{array}$ \\
\hline
\end{tabular}

Fonte: Elaborado pelos autores.

Quanto ao tipo de estudo dos artigos, foi identificado um observacional, um longitudinal, um de abordagem mista e os demais ( $n$ ) foram transversais. Ademais, observou-se nos estudos uma predominância de estudantes do sexo feminino, sendo avaliados de 152 a 10751 acadêmicos de medicina.

\section{Discussão}

O declínio da empatia em estudantes de Medicina vem sendo percebido em todo o mundo, tornando importante o entendimento dos fatores responsáveis por este fenômeno. Diversos estudos evidenciaram que os anos de graduação influenciam significativamente, seja de forma positiva8,24 ou negativa ${ }^{13,15,19,25}$ no escore global de empatia. Por outro lado, houve estudos que não identificaram nenhuma relação significativa ${ }^{14,18,21,22}$. Outros três estudos não investigaram a relação da empatia com os anos de graduação em medicina ${ }^{12,23,27}$.

Os demais estudos, no total quatro, analisaram a empatia comparada aos anos da graduação e obtiveram desfechos particulares. Em um estudo com acadêmicos da Índia, verificou-se que os escores médios de empatia diminuíram do primeiro para o terceiro semestre, mas voltaram a subir no sétimo semestre ${ }^{17}$. Uma pesquisa que analisou a empatia em estudantes chineses identificou que as pontuações aumentaram até o quinto ano, mantiveram-se estáveis no sexto e diminuíram a partir do sétimo ano da graduação de um curso de oito $\operatorname{anos}^{20}$. Na pesquisa com universidades portuguesas, na universidade com maior escore médio de empatia, os alunos do terceiro ano apresentaram as maiores pontuações ${ }^{26}$. Já em outro estudo com estudantes da América do Sul, observou-se uma redução da empatia ao longo da graduação em uma universidade do Equador e um aumento do escore em uma universidade da Colômbia ${ }^{16}$.

A fim de se entender como as características pessoais de cada indivíduo influenciam em sua atitude empática, alguns aspectos se destacam, como as variáveis gênero e idade. Quanto ao gênero, vários estudos analisados concluíram que mulheres tendem a ser mais empáticas $8,12,14,15,17,21,23-26$. Por outro lado, em um estudo realizado na Irlanda, os homens foram identificados com maiores escores de empatia ${ }^{18}$, e nos demais estudos não se identificou uma relação significativa entre empatia e gênero ${ }^{13,16,19,20,22,27}$.

No que se refere à idade, por sua vez, uma pesquisa com estudantes de medicina da Índia concluiu que a empatia não foi significativamente associada à idade ${ }^{17}$. Por outro lado, em estudantes de medicina da África do $\mathrm{Sul}^{23}$ e do Brasili ${ }^{8}$, identificou-se que essas duas variáveis estão significativamente associadas, percebendo-se maiores escores de empatia em idades menores e em idades maiores, respectivamente.

Em contrapartida, a razão para a mudança emocional e cognitiva da empatia nesses indivíduos pode estar relacionada a fatores externos, como a formação acadêmica. Nesse sentido, é importante avaliar a metodologia empregada pelas instituições, além de suas matrizes curriculares. Em um estudo comparando os acadêmicos de duas faculdades de medicina portuguesas, encontraram-se escores de empatia maiores naquela cujo sistema de ensino é integrado, a grade curricular apresenta mais conteúdos de ciências humanas e, por fim, na qual o contato prático com os pacientes é mais precoce ${ }^{26 .}$

O efeito positivo do contato clínico mais precocemente, real ou simulado, foi identificado por uma pesquisa com estudantes 
de medicina na China ${ }^{27}$. Concluíram que os estudantes chineses do primeiro e segundo ano apresentaram melhoria no escore de empatia após terem sido submetidos a um programa de duas semanas de"encontro clínico precoce" ${ }^{\prime 27}$. No entanto, outro estudo, também com acadêmicos chineses, identificou que a empatia diminuía consideravelmente nos últimos dois anos do curso, período no qual se inicia a prática e o treinamento clínico propriamente dito ${ }^{20}$.

Além da prática clínica, as escolhas ou preferências dos estudantes de medicina também podem alterar e predizer sua tendência a ser mais ou menos empático. Tendo isso em vista, estudos encontraram diferença significativa no escore de empatia de acordo com a preferência de carreira futura. Concluiu-se que os estudantes que preferiam ou optavam por especialidades clínicas, nas quais a relação médico-paciente é muito mais frequente, tiveram pontuações maiores do que aqueles que preferiam as especialidades cirúrgicas ou voltadas para tecnologias ${ }^{15,20,22,25}$. Em contrapartida, há também estudos nos quais essa relação não foi estatisticamente significante ${ }^{13,19}$.

Além disso, vale ressaltar outras variáveis que foram analisadas. Em um estudo com acadêmicos de medicina da cidade de Goiânia, Goiás, Brasil, ficou demonstrado que a participação em trabalhos voluntários e contato próximo com enfermidade de amigos são fatores relacionados com maiores escores de empatia ${ }^{8}$. Ademais, o fato de cursarem medicina como sendo a primeira opção de carreira do estudante também é um preditor de maiores escores de empatia ${ }^{12,20}$.

Em adição, um estudo realizado na Universidade de Cartagena analisou a influência das características familiares e demonstrou que os estudantes cujas famílias não tinham nenhuma disfuncionalidade apresentaram maiores níveis de empatia, permitindo inferir a importância da família e da relação entre seus membros no desenvolvimento das habilidades de interação ${ }^{12}$. Ademais, o desempenho acadêmico também deve ser levado em consideração, uma vez que alunos que apresentaram maiores notas, revelaram maior nível de empatia ${ }^{12}$. No entanto, um estudo não encontrou relação significativa entre o desempenho acadêmico e os escores de empatia ${ }^{20}$.

Em última análise, estudos avaliaram os elementos da empatia e quais deles sofrem maiores alterações durante o curso. Um estudo brasileiro demonstrou que as variáveis afetivas são as mais afetadas durante a graduação médica ${ }^{21}$. Além disso, dois estudos apontaram que o fator que mais se altera no decorrer da graduação é o "cuidado compassivo" 8,12 , enquanto outros dois apresentaram o fator "tomada de perspectiva"16,26. Por outro lado, em um dos estudos não foi encontrado alteração significativa das dimensões que compõem a Escala Jefferson de Empatia ao longo do tempo ${ }^{25}$.

\section{Conclusão}

Sabendo-se da influência positiva da empatia sobre a relação médico-paciente, estudos acerca do tema vêm sendo realizados em todo o mundo. Os estudos analisados nesta revisão, os quais avaliaram a empatia em estudantes de medicina por meio da Escala Jefferson de Empatia (versão para estudantes), apontaram variáveis próprias do indivíduo e também variáveis externas, principalmente relacionadas à sua formação acadêmica, que podem interferir na atitude empática diante um encontro clínico.

Nesse sentido, a partir dessa revisão, quanto às características pessoais do indivíduo, percebeu-se uma tendência de maiores escores de empatia em estudantes jovens, do sexo feminino, com maior desempenho acadêmico e que almejam seguir especialidades clínicas no futuro. Já quanto às variáveis externas, início do curso e grade curricular composta por mais conteúdos de ciências humanas e com encontro clínico mais precoce apresentam influência positiva na atitude empática. Por fim, as dimensões afetivas foram as que mais sofreram alteração.

Diante das evidências encontradas é possível entender como a empatia nos estudantes de medicina é moldada por diversas variáveis durante sua formação acadêmica. Isso permite desenvolver estratégias a fim de se solucionar a tendência decrescente dos escores de empatia ao longo da graduação, por meio da introdução, principalmente, de encontro clínico mais precoce e de matérias das ciências humanas nas grades curriculares em cursos de medicina em todo o mundo.

Mais análises e estudos sobre este tema devem ser desenvolvidos a fim de, cada vez mais, entender como a empatia pode ser aperfeiçoada durante a graduação. Assim, a boa relação médico-paciente se tornará mais frequente na prática clínica e trará benefícios mútuos para o paciente e para o médico.

\section{Referências}

1. Machado CDB, Wuo A, Heinzle M. Educação médica no Brasil: uma análise histórica sobre a formação acadêmica e pedagógica. Rev Bras Educ Med. 2018;42(4):66-73.

2. Conselho Nacional De Educação. Diretrizes Curriculares Nacionais do Curso de Graduação em Medicina, 2001. 2001;1-6.

3. Conselho Nacional De Educação. Diretrizes Curriculares Nacionais do Curso de Graduação em Medicina, 2014. 2014;2014(c):8-11.

4. Meireles MAC, Fernandes CCP, Silva LS. Novas diretrizes curriculares nacionais e a formação médica: expectativas dos discentes do primeiro ano do curso de medicina de uma instituição de ensino superior. Rev Bras Educ Med. 2019;43(2):67-78.

5. Pires MFDN, Roazzi A. Empatia e sua avaliação: con- 
siderações teóricas e metodológicas. Rev Amaz. 2016;17(1):158-72.

6. Oliveira NC, Bandeira S, Pitanga AV. O conceito de empatia sob a perspectiva da psicologia contemporânea. Centro Universitário de Anápolis - UniEvangélica. 2019;1-16.

7. Mufato LF, Gaíva MAM. Empatia em saúde: revisão integrativa. Rev Enferm do Cent Oeste Min. 2019;9:1-12.

8. Nascimento HCF, Ferreira Júnior WA, Silva AMTC, Carvalho IGM, Bastos GCFC, Almeida RJ. Análise dos níveis de empatia de estudantes de medicina. Rev Bras Educ Med. 2018;42(1):152-60.

9. Charlo JCP, Dios MTC. Empatia no cotidiano do curso de graduação de medicina a partir de uma revisão integratva. Brazilian J Dev. 2019;5(6):5983-92.

10. Souza MT, Silva MD, Carvalho R. Revisão integrativa: o que é e como fazer. Einstein. 2009;8(1):102-6.

11. Moher D, Liberati A, Tetzlaff J, Altman DG, Altman D, Antes $G$, et al. Preferred reporting items for systematic reviews and meta-analyses: The PRISMA statement. PLoS Med. 2009;6(7):e1000097.

12. Anaya MVM, Amador LRT, Martínez FG. Factores relacionados con la empatía en estudiantes de medicina de la Universidad de Cartagena. Rev Clin Med Fam. 2015;8(3):185-92.

13. Benabbas R. Empathy in Iranian medical students: a comparison by age, gender, academic performance and specialty preferences. Med J Islam Repub Iran. 2016;30(1):1-7.

14. QuinceTA, Kinnersley P, Hales J, Silva A, Moriarty H, Thiemann $\mathrm{P}$, et al. Empathy among undergraduate medical students: a multi-centre cross-sectional comparison of students beginning and approaching the end of their course. BMC Med Educ. 2016;16(1):1-10.

15. Raof AM, Yassin BA. Measuring empathy levels among Kurdish medical students in Erbil City, Iraq cross-sectional study. Sultan Qaboos Univ Med J. 2016;16(1):e62-7.

16. Calzadilla-Núñez A, Díaz-Narváez VP, Dávila-Pontón $Y$, Aguilera-Muños J, Fortich-Mesa N, Aparicio-Marenco $\mathrm{D}$, et al. Erosión empática durante la formación médica según el género. estudio transversal. Arch Argent Pediatr. 2017;115(6):556-61.

17. Chatterjee A, Ravikumar R, Singh S, Chauhan OS, Goel M. Clinical empathy in medical students in India measured using the Jefferson Scale of Empathy-Student Version. J Educ Eval Health Prof. 2017;14(33):1-6.

18. O'Sullivan DM, Moran J, Corcoran P, O'Flynn S, O'Tuathaigh C, O'Sullivan AM. Medical school selection criteria as predictors of medical student empathy: a cross-sectional study of medical students, Ireland. BMJ Open. 2017;7:e016076.

19. Tariq N, Rasheed T, Tavakol M. A quantitative study of empathy in pakistani medical students: A multicentered approach. J Prim Care Community Heal. 2017;8(4):294-9.

20. Li D, Xu H, Kang M, Ma S. Empathy in chinese eight-year medical program students: differences by school year, educational stage, and future career preference. BMC Med Educ. 2018;18(1):1-9.

21. Moreto G, Santos IS, Pessini L, Lotufo PA. Assessing empathy among medical students: A comparative analysis using two different scales in a brazilian medical school. Educ Médica. 2018;19:162-70.

22. Tariq N, Tayyab A, Jaffery T. Differences in empathy levels of medical students based on gender, year of medical school and career choice. J Coll Physicians Surg Pakistan. 2018;28(4):310-3.

23. Archer $E$, Turner R. Measuring empathy in a group of South African undergraduate medical students using the student version of the Jefferson Scale of Empathy. African J Prim Heal Care Fam Med. 2019;11(1):1-5.

24. Ulloque MJ, Villalba S, de Villalba TV, Fantini A, Quinteros S, Díaz-Narváez V. Niveles de empatía en estudiantes de medicina de Córdoba, Argentina. Arch Argent Pediatr. 2019;117(2):81-6.

25. Hojat M, Shannon SC, Desantis J, Speicher MR, Bragan $\mathrm{L}$, Calabrese LH. Does empathy decline in the clinical phase of medical education? a nationwide, multiinstitutional, cross-sectional study of students at DOGranting Medical Schools. Acad Med. 2020;95(6):911-8.

26. Santiago LM, Rosendo I, Coutinho ML, Maurício KS, Neto I, Simões JA. Comparing empathy in medical students of two Portuguese medicine schools. BMC Med Educ. 2020;20(1):1-6.

27. Ye X, Guo H, Xu Z, Xiao H. Empathy variation of undergraduate medical students after early clinical contact: a cross-sectional study in China. BMJ Open. 2020;10(7):e035690. 